\title{
Evaluation of variation in the phosphoinositide-3-kinase catalytic subunit alpha oncogene and breast cancer risk
}

KN Stevens', M Garcia-Closas', Z Fredericksen', M Kosel', VS Pankratz', JL Hopper ${ }^{3}$, GS Dite ${ }^{3}$, C Apicella $^{3}$, MC Southey ${ }^{4}$, MK Schmidt ${ }^{5}$, A Broeks ${ }^{5}$, LJ Van 't Veer ${ }^{5}$, RAEM Tollenaar', PA Fasching, ${ }^{7}$, MW Beckmann $^{8}$,

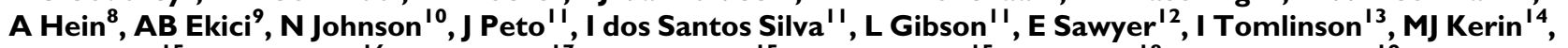
S Chanock ${ }^{15}$, J Lissowska ${ }^{16}$, DJ Hunter ${ }^{17}$, RN Hoover ${ }^{15}$, GD Thomas ${ }^{15}$, RL Milne ${ }^{18}$, Jl Arias Pérez ${ }^{19}$, A González-Neira ${ }^{20}$, J Benítez ${ }^{21}$, B Burwinkel ${ }^{22,23}$, A Meindl $^{24}$, RK Schmutzler ${ }^{25}$, CR Bartrar ${ }^{26}$, U Hamann ${ }^{27}$, YD Ko ${ }^{28}$, T Brüning ${ }^{29}$, J Chang-Claude ${ }^{30}$, R Hein ${ }^{30}$, S Wang-Gohrke ${ }^{31}$, T Dörk ${ }^{32}$, P Schürmann ${ }^{32}$, M Bremer $^{33}$, P Hillemanns $^{34}$, N Bogdanova ${ }^{33}$, JV Zalutsky ${ }^{34}$, YI Rogov ${ }^{34}$, N Antonenkova ${ }^{34}$, A Lindblom ${ }^{35}$, 5 Margolin ${ }^{36}$, A Mannermaa ${ }^{37}$, V Kataja ${ }^{38}$, V-M Kosma ${ }^{37}$, J Hartikainen ${ }^{37}$, G Chenevix-Trench ${ }^{39}$, X Chen ${ }^{39}$, P Peterlongo ${ }^{40}$, B Bonanni ${ }^{41}$, L Bernard ${ }^{42}$, S Manoukian ${ }^{43}$, X Wang', J Cerhan', CM Vachon', J Olson', GG Giles ${ }^{44,45}$, L Baglietto ${ }^{4,45}$, CA McLean ${ }^{46}$, G Severi ${ }^{44,45}$, EM John ${ }^{47}$, A Miron ${ }^{48}$, R Winqvist ${ }^{49}$, K Pylkäs ${ }^{49}$, A Jukkola-Vuorinen ${ }^{50}$, M Grip ${ }^{51}$, I Andrulis ${ }^{52,53,54}$, JA Knight ${ }^{55,56}$, G Glendon ${ }^{52}$, AM Mulligan ${ }^{57,58}$, A Cox ${ }^{59}$, IW Brock ${ }^{59}$, G Elliott ${ }^{60}$, SS Cross ${ }^{61}$, PP Pharoah ${ }^{62}$, AM Dunning ${ }^{63}$, KA Pooley S $^{63}$, MK Humphreys ${ }^{64}$, J Wang ${ }^{64}$, D Kang ${ }^{65}$, K-Y Yoo ${ }^{65}$, D-Y Noh ${ }^{65}$, S Sangrajrang ${ }^{66}$, V Gabrieau ${ }^{67}$, P Brennan ${ }^{67}$, J McKay ${ }^{67}$, H Anton-Culver ${ }^{68}$, A Ziogas ${ }^{68}$, FJ Couch ${ }^{*, 69}$ and DF Easton ${ }^{63,64}$ the GENICA Network, kConFab Investigators, Australian Ovarian Cancer Study Group ${ }^{70}$

'Department of Health Sciences Research, Mayo Clinic, 200 First Street SW, Rochester, MN 55905, USA; ${ }^{2}$ Sections of Epidemiology and Genetics, Institute of Cancer Research and Breakthrough Breast Cancer Research Centre, 123 Old Brompton Road, London SW7 3RP, UK; ${ }^{3}$ Centre for Molecular, Environmental, Genetic and Analytic Epidemiology, The University of Melbourne, Victoria 30 I0, Australia; ${ }^{4}$ Department of Pathology, The University of Melbourne, Victoria 30 10, Australia; ${ }^{5}$ The Netherlands Cancer Institute, Plesmanlaan 121, 1066 CX. Amsterdam, The Netherlands; ${ }^{6}$ Leiden University Medical Center, Albinusdreef 2, 2333 ZA, Leiden, The Netherlands; ${ }^{7}$ Division of Hematology and Oncology, Department of Medicine, University of California at Los Angeles, David Geffen School of Medicine, Los Angeles, CA 90095, USA; ${ }^{8}$ Department of Gynecology and Obstetrics, University Breast Center Franconia, Friedrich-Alexander University Erlangen-Nuremberg, Comprehensive Cancer Center Erlangen-Nuremberg, Schloßplatz 4, 91054 Erlangen, Germany; ${ }^{9}$ Institute of Human Genetics, Friedrich-Alexander University Erlangen-Nuremberg, Schloßplatz 4, 9 I 054 Erlangen, Germany; ${ }^{10}$ Breakthrough Breast Cancer Research Centre, Institute of Cancer Research, 123 Old Brompton Road, London SW7 3RP, UK; "'Faculty of Epidemiology and Population Health, London School of Hygiene and Tropical Medicine, Keppel Street, London WCIE 7HT, UK; ${ }^{12}$ National Institute for Health Research (NIHR) Comprehensive Biomedical Research Centre, Guy's and St Thomas' NHS Foundation Trust in partnership with King's College London and King's College Hospital NHS Foundation Trust, Guy's Hospital, Great Maze Pond, London SE I 9RT, UK; '13 Oxford Biomedical Research Centre, Molecular and Population Genetics, Wellcome Trust Centre for Human Genetics, University of Oxford, Oxford OX3 7BN, UK; ${ }^{14}$ NUIG Department of Surgery, Clinical Science Institute, University Hospital Galway, Galway 091524 4I I, Ireland; ${ }^{15}$ Division of Cancer Epidemiology and Genetics, National Cancer Institute, 61 20 Executive Boulevard, MSC 7242, Bethesda, MD 20892-7335, USA; ${ }^{16}$ Department of Cancer Epidemiology and Prevention, M. Sklodowska-Curie Memorial Cancer Center, Institute of Oncology, 5 Roentgena Street, 02-78I Warsaw, Poland; ${ }^{17}$ Program in Molecular and Genetic Epidemiology, Harvard School of Public Health, 677 Huntington Avenue, Boston, MA 021 I 5, USA; ${ }^{18}$ Genetic and Molecular Epidemiology Group, Spanish National Cancer Research Centre (CNIO), Calle Melchor Fernández Almagro 3, Madrid 28029, Spain; ${ }^{19}$ Servicio de Cirugía General y Especialidades, Hospital Monte Naranco, Av. Doctores Fernández Vega 107, Oviedo 330 12, Spain; ${ }^{20}$ Human Gentyping Unit (CeGen), Spanish National Cancer Research Centre (CNIO), Madrid, Spain; ${ }^{2}$ Human Genetics Group, Spanish National Cancer Research Centre (CNIO), Calle Melchor Femández Almagro 3, Madrid 28029, Spain; ${ }^{22}$ Department of Obstetrics and Gynecology, University Hospital Heidelberg, Im Neuenheimer Feld 280, D-69I 20 Heidelberg, Germany; ${ }^{23}$ Molecular Epidemiology Group, German Cancer Research Center (DKFZ), Im Neuenheimer Feld 280, D-69I 20 Heidelberg, Germany; ${ }^{24}$ Department of Gynaecology and Obstetrics, Technical University of Munich, Arcisstraße 21, 80333 Munich, Germany; ${ }^{25}$ Division of Molecular GynecoOncology, Department of Gynaecology and Obstetrics, Center of Molecular Medicine Cologne (CMMC), University Hospital of Cologne, 5093 I Cologne, Germany; ${ }^{26}$ Institute of Human Genetics, University of Heidelberg, Im Neuenheimer Feld 366, 69I 20 Heidelberg, Germany; ${ }^{27}$ Deutsches Krebsforschungszentrum (DKFZ), Im Neuenheimer Feld 280, D-691 20 Heidelberg, Germany; ${ }^{28}$ Department of Internal Medicine, Evangelische Kliniken Bonn gGmbH, Betriebsstätte Johanniter Krankenhaus, Johanniterstraße 3-5; 531 I 3 Bonn, Germany; ${ }^{29}$ Institute for Prevention and Occupational Medicine of the German Social Accident Insurance (IPA), Burkle-de-la-Camp-Platz I, 44789 Bochum, Germany; ${ }^{30}$ Division of Cancer Epidemiology, German Cancer Research Center (DKFZ), Im Neuenheimer Feld 280, D-691 20 Heidelberg, Germany; ${ }^{31}$ Department of Obstetrics and Gynecology, University of Ulm, Albert-Einstein-Allee II, 8908 I Ulm, Germany; ${ }^{32}$ Hannover Medical School, Gynaecology Research Unit, 30625 Hannover, Germany; ${ }^{33}$ Hannover Medical School, Clinics of Radiation Oncology, 30625 Hannover, Germany; ${ }^{34}$ Hannover Medical School, Clinics of Obstetrics and Gynaecology, 30625 Hannover, Germany; ${ }^{35}$ Department of Molecular Medicine and Surgery, Karolinska Institutet, SI 7176 Stockholm, Sweden; ${ }^{36}$ Department of Oncology Pathology, Karolinska Institutet, SI 7176 Stockholm, Sweden; ${ }^{37}$ Institute of Clinical Medicine, Department of Pathology, University of Eastern Finland and Kuopio University Hospital, Biocenter Kuopio, Fl-702 I I Kuopio, Finland; ${ }^{38}$ Institute of Clinical Medicine, Department of Oncology, University of Eastern Finland, Kuopio University Hospital, Fl-7021 I Kuopio, Finland; ${ }^{39}$ Queensland Institute of Medical Research, 300 Herston Road, Herston, QLD 4029,

*Correspondence: Dr FJ Couch; E-mail: couch.fergus@mayo.edu

${ }^{70}$ See Appendix.

Received I2 July 20 I ; revised I6 September 20 I ; accepted 28 September 20 II; published online 27 October 20I I 
Australia; ${ }^{40}$ Unit of Molecular Bases of Genetic Risk and Genetic Testing, Department of Preventive and Predicted Medicine, Fondazione IRCCS Istituto Nazionale Tumori (INT) and IFOM, Fondazione Istituto FIRC di Oncologia Molecolare; via Adamello, 16 - 20139 Milano, Italy; ${ }^{41}$ Division of Cancer Prevention and Genetics, Istituto Europeo di Oncologia, via Ripamonti 435, 20141 Milano, Italy; ${ }^{42}$ Department of Experimental Oncology, Istituto Europeo di Oncologia and Consortium for Genomics Technology (Cogentech); via Adamello, 16 - 20139 Milano, Italy; ${ }^{43}$ Unit of Medical Genetics, Department of Preventive and Preventive Medicine, Fondazione IRCCS Istituto Nazionale Tumori (INT), via Venezian I - 20133 Milano, Italy; ${ }^{44}$ Cancer Epidemiology Centre, Cancer Council Victoria, I Rathdowne Street, Carlton VIC 3053, Australia; ${ }^{45}$ Centre for Molecular, Environmental, Genetic, and Analytic Epidemiology, The University of Melboume, Level 4, 207 Bouverie Street, Victoria 30 I 0, Australia; ${ }^{46}$ Department of Anatomical Pathology, The Alfred Hospital, Commercial Road, Prahran VIC 3181, Australia; ${ }^{47}$ Department of Epidemiology, Cancer Prevention Institute of California, 2201 Walnut Avenue, Suite 300, Fremont, CA 94538, USA; ${ }^{48}$ Department of Surgery, Dana-Farber Cancer Institute, 450 Brookline Avenue, Boston, MA 022I 5-5450, USA; ${ }^{49}$ Laboratory of Cancer Genetics, Department of Clinical Genetics and Biocenter Oulu, University of Oulu, Oulu University Hospital, Aapistie 5 A, 90220 Oulu, Finland; ${ }^{50}$ Department of Oncology, University of Oulu, Oulu University Hospital, Aapistie 5 A, 90220 Oulu, Finland; ${ }^{51}$ Department of Surgery, University of Oulu, Oulu University Hospital, Aapistie 5 A, 90220 Oulu, Finland; ${ }^{52}$ Ontario Cancer Genetics Network, Cancer Care Ontario, 620 University Avenue, Toronto, ON M5G 2CI ON, Canada; ${ }^{53}$ Fred A. Litwin Center for Cancer Genetics, Mount Sinai Hospital, 600 University Avenue, Toronto, ON M5G IX5, ON Canada; ${ }^{54}$ Department of Molecular Genetics, University of Toronto, 105 George Street, Toronto, ON M5A 2N4 ON, Canada; ${ }^{55}$ Samuel Lunenfeld Research Institute, Mount Sinai Hospital, 600 University Avenue, Toronto, ON M5G IX5 ON, Canada; ${ }^{56}$ Division of Epidemiology, Dalla Lana School of Public Health, University of Toronto, 105 George Street, Toronto, ON M5A 2N4 ON, Canada; ${ }^{57}$ Department of Laboratory Medicine and Pathobiology, University of Toronto, 105 George Street, Toronto, ON M5A 2N4 ON, Canada; ${ }^{58}$ Department of Laboratory Medicine and Keenan Research Centre of the Li Ka Shing Knowledge Institute, St Michael's Hospital, 30 Bond Street, Toronto, ON M5B I W8 ON, Canada; ${ }^{59}$ Faculty of Medicine, Dentistry and Health, University of Sheffield, Beech Hill Road, Sheffield SIO2RX, UK; ${ }^{60}$ Medical Genetics Research Group, Faculty of Medicine and Human Sciences, University of Manchester, Oxford Road, Manchester MI3 9PL, UK; ${ }^{6}$ Department of Neuroscience, Faculty of Medicine, Dentistry and Health, University of Sheffield, Beech Hill Road, Sheffield SI 02RX, UK; ${ }^{62}$ United Kingdom Department of Oncology and Department of Public Health and Primary Care University of Cambridge, Cambridge, UK; ${ }^{63}$ Department of Oncology, University of Cambridge, Robinson Way, Cambridge CB2 ORE, UK; ${ }^{64}$ Centre for Cancer Genetic Epidemiology, University of Cambridge, Worts Causeway, Cambridge CBI 8RN, UK; ${ }^{65}$ Seoul National University College of Medicine, Yongon _ 103 Daehak-ro, Jongno-gu, Seoul I I 0-799, Korea; ${ }^{66}$ National Cancer Institute, Rama 6 Road, 10400 Bangkok, Thailand; ${ }^{67}$ International Agency for Research on Cancer, 150 Cours Albert Thomas, 69372 Lyon CEDEX 08, France;

${ }^{68}$ Department of Epidemiology, School of Medicine, University of California Irvine, 224 Irvine Hall, Irvine, CA 92697, USA; ${ }^{69}$ Department of Laboratory Medicine and Pathology, Mayo Clinic, 200 First Street SW, Rochester, MN 55905, USA

BACKGROUND: Somatic mutations in phosphoinositide-3-kinase catalytic subunit alpha (PIK3CA) are frequent in breast tumours and have been associated with oestrogen receptor (ER) expression, human epidermal growth factor receptor-2 overexpression, lymph node metastasis and poor survival. The goal of this study was to evaluate the association between inherited variation in this oncogene and risk of breast cancer.

METHODS: A single-nucleotide polymorphism from the PIK3CA locus that was associated with breast cancer in a study of Caucasian breast cancer cases and controls from the Mayo Clinic (MCBCS) was genotyped in 5436 cases and 5280 controls from the Cancer Genetic Markers of Susceptibility (CGEMS) study and in 30949 cases and 29788 controls from the Breast Cancer Association Consortium (BCAC).

RESULTS: Rs 1607237 was significantly associated with a decreased risk of breast cancer in MCBCS, CGEMS and all studies of white Europeans combined (odds ratio $(\mathrm{OR})=0.97,95 \%$ confidence interval $(\mathrm{Cl}) 0.95-0.99, \mathrm{P}=4.6 \times 10^{-3}$ ), but did not reach significance in the $B C A C$ replication study alone $(O R=0.98,95 \% \mathrm{Cl} 0.96-1.01, P=0.139)$.

CONCLUSION: Common germline variation in PIK3CA does not have a strong influence on the risk of breast cancer British Journal of Cancer (201I) 105, 1934-1939. doi:10.1038/bjc.2011.448 www.bjcancer.com

Published online 27 October 2011

(c) 201 I Cancer Research UK

Keywords: genetic susceptibility; neoplasms; association study

Phosphatidylinositol-3 kinases (PI3Ks) constitute a lipid kinase family integral to signalling pathways that regulate many cancerrelated processes, including cell proliferation, adhesion, apoptosis, survival and motility (Fruman et al, 1998; Cantley, 2002). Alteration of PI3K family members, such as amplification of the phosphoinositide-3-kinase catalytic subunit alpha (PIK3CA) oncogene on chromosome $3 \mathrm{q} 26$ that encodes the $\mathrm{p} 110 \alpha$ catalytic subunit of PI3K, are commonly observed in human cancers. Amplification and overexpression of PIK3CA results in increased production of the phosphatidylinositol-3,4,5-triphosphate second messenger, hyperactivation of the PI3K/AKT pathway, and stimulation of cellular transformation and tumour progression (Shayesteh et al, 1999; Ma et al, 2000; Fresno Vara et al, 2004; Saal et al, 2005; Samuels and Ericson, 2006). Somatic mutations in PIK3CA are also common in colon $(18-32 \%)$, gastric $(4-25 \%)$, endometrial (36\%), liver (36\%), brain (27\%) and breast (18-40\%) tumours (Bachman et al, 2004; Campbell et al, 2004; Samuels et al, 2004; Karakas et al, 2006; Ligresti et al, 2009). Functional analyses have shown that many of these mutations activate PIK3CA enzymatic activity and stimulate downstream AKT signalling, promoting growth factor-independent growth and metastasis (Samuels et al, 2004; Samuels and Ericson, 2006).

In breast tumours, PIK3CA mutations have been consistently associated with ER-positive and human epidermal growth factor receptor-2 (HER2)-positive tumour status (Saal et al, 2005; Li et al, 2006; Perez-Tenorio et al, 2007; Stemke-Hale et al, 2008) (Saal et al, 2005; Perez-Tenorio et al, 2007). The correlation between these mutations and breast cancer prognosis is less clear, with several studies reporting associations between PIK3CA mutations and lymph node metastasis and worse overall and breast cancer-specific survival (Saal et al, 2005; Li et al, 2006; Lai et al, 2008; Aleskandarany et al, 2010), whereas other studies have 
reported associations with longer survival particularly among patients with ER-positive, HER2-negative tumours (Perez-Tenorio et al, 2007; Kalinsky et al, 2009; Loi et al, 2010).

Although the pathological and clinical significance of PIK3CA somatic mutations has been well studied, the contribution of inherited variation in this important oncogene to risk of breast cancer is unknown. Here we investigated the influence of germline variation in PIK3CA on breast cancer risk.

\section{MATERIALS AND METHODS}

\section{Mayo clinic breast cancer study}

The details of the Mayo Clinic Breast Cancer case-control Study (MCBCS) have been described previously (Wang et al, 2008). Briefly, cases were comprised of Caucasian women with invasive breast cancer diagnosed within 6 months of ascertainment with no prior history of cancer. Controls were comprised of Caucasian women visiting the Mayo Clinic for general medical exams in the Department of Internal Medicine with no prior history of cancer. Participants were recruited under an Institutional Review Board approved protocol. A total of 798 cases and 843 controls were utilised for stage 1 genotyping (Table 1 ).

\section{Replication studies}

The Cancer Genetic Markers of Susceptibility (CGEMS) breast cancer case-control study and 26 case-control studies from Breast Cancer Association Consortium (BCAC) contributed data to these analyses (described in Supplementary Table 1). Stage 1 of the CGEMS GWAS included 1145 cases and 1142 controls of selfreported white European ancestry (Thomas et al, 2009), whereas the combined Stage 1 and 2 of CGEMS included a total of 5436 cases and 5280 controls (Table 1). The BCAC replication was comprised of 24 studies of women of primarily European descent (Supplementary Table 1), 1702 additional samples from MCBCS and two studies (SEBCS and TBCS) of women from Southeast Asia (Table 1). Final combined analyses included 35991 breast cancer cases and 35153 controls of white European ancestry, as well as 2183 breast cancer cases and 1469 controls of Asian ancestry. Study participants were recruited under protocols approved by the institutional review board at each institution and all subjects provided written informed consent.

\section{Genotyping}

Four haplotype-tagging single-nucleotide polymorphisms (SNPs) within PIK3CA (rs13320527, rs3729692, rs1607237, rs9838117) were selected $\left(r^{2}>0.80\right.$ in European-American genotype data from HapMap release 21). A total of 1741 Mayo Clinic samples (798 cases, 843 controls and 100 duplicates) were genotyped on custom oligo pool assays at Illumina Corporation (San Diego, CA, USA) using the Illumina GoldenGate assay. All SNPs had genotype call rates $>95 \%$. Concordance between duplicate samples was $100 \%$. Genotyping of rs1607237 in CGEMS and BCAC was performed using a TaqMan allelic discrimination assay or the Sequenom platform (Sequenom, San Diego, CA, USA) via standard protocols. Genotyping concordance was verified with internal duplicates and overall data quality was ensured using independent genotyping of $96 \mathrm{CEU}$ samples by each genotyping center (Garcia-Closas et al, 2008). All studies met the specified criteria for call rate $(>95 \%)$.

\section{Pathology and tumour markers}

The collection of pathology and tumour marker information for $\mathrm{BCAC}$ has been described previously (Yang et al, 2011). Pathology data were also available for 900 CGEMS subjects. Briefly, studies provided information on histopathological subtype, grade of
Table I Studies contributing to evaluation of associations between rs 607237 and breast cancer risk

\begin{tabular}{|c|c|c|c|}
\hline Study ${ }^{a}$ & Country & Cases $n(\%)$ & Controls n (\%) \\
\hline ABCFS & Australia & $1199(3.1)$ & $438(1.2)$ \\
\hline $\mathrm{ABCS}$ & The Netherlands & | $465(3.8)$ & $548(1.5)$ \\
\hline $\mathrm{BBCC}$ & Germany & $1060(2.8)$ & $994(2.7)$ \\
\hline BBCS & UK & $1153(3.0)$ & $831(2.3)$ \\
\hline BIGGS & Ireland & $1060(2.8)$ & $900(2.5)$ \\
\hline CGEMS $^{b}$ & USA & $5436(14.2)$ & $5280(14.4)$ \\
\hline CNIO-BCS & Spain & $752(2.0)$ & $823(2.2)$ \\
\hline GC-HBOC & Germany & $864(2.3)$ & $1224(3.3)$ \\
\hline GENICA & Germany & $1013(2.7)$ & $1012(2.8)$ \\
\hline GESBC & Germany & $563(1.5)$ & $564(1.5)$ \\
\hline HABCS & Germany & $1046(2.7)$ & $998(2.7)$ \\
\hline HMBCS & Belarus & $1760(4.6)$ & $1015(2.8)$ \\
\hline KARBAC & Sweden & $812(2.1)$ & $863(2.4)$ \\
\hline kConFab/AOCS & Australia/New Zealand & $566(1.5)$ & $899(2.5)$ \\
\hline $\mathrm{KBCP}$ & Finland & $485(1.3)$ & $427(1.2)$ \\
\hline MARIE & Germany & $2754(7.2)$ & $5302(14.5)$ \\
\hline MBCSG & Italy & $739(1.9)$ & $123 \mid(3.4)$ \\
\hline $\mathrm{MCBCS}^{\mathrm{C}}$ & USA & $1789(4.7)$ & $1554(4.2)$ \\
\hline MCCS & Australia & $679(1.8)$ & $751(2.1)$ \\
\hline NC-BCFR & USA & $388(1.0)$ & $154(0.4)$ \\
\hline OBCS & Finland & $544(1.4)$ & $509(1.4)$ \\
\hline OFBCR & Canada & $1170(3.1)$ & $329(0.9)$ \\
\hline SBCS & UK & $1217(3.2)$ & $120 \mid(3.3)$ \\
\hline SEARCH & UK & $6520(17.1)$ & $6779(18.5)$ \\
\hline SEBCS $^{d}$ & Korea & | $732(4.5)$ & I 178 (3.2) \\
\hline $\mathrm{TBCS}^{\mathrm{d}}$ & Thailand & $451(1.2)$ & $29 \mid(0.8)$ \\
\hline UCIBCS & USA & $957(2.5)$ & $527(1.4)$ \\
\hline Total & & $38174(100)$ & $36622(100)$ \\
\hline
\end{tabular}

aSee Supplementary Table I for definition of study acronyms. ${ }^{b}$ Stage 2: Cancer Genetic Markers of Susceptibility study. 'Includes Stage I: Mayo Clinic Breast Cancer Study. ${ }^{\mathrm{d}}$ Asian case-control studies.

differentiation, tumour size, nodal involvement and stage at diagnosis of breast tumours. All studies except BBCS, GC-HBOC and HMBCS provided data on ER and progesterone receptor (PR) status of tumours, and 12 studies provided data on HER2 (Supplementary Table 2). ER/PR status was most commonly defined using data from medical records. Oestrogen receptor and PR negative status was defined as $<10 \%$ of the tumour cells stained. Human epidermal growth factor receptor-2-negative status was typically defined as a score of 0 or $1+$ on a HER2 immunohistochemistry (IHC) scale of $0-3+$.

\section{Statistical methods}

Evidence of departure from Hardy-Weinberg equilibrium (HWE) was assessed in controls using a goodness of fit test and none was observed (HWE $P \geqslant 0.001$ ). Single-nucleotide polymorphism associations were tested using unconditional logistic regression adjusting for age and state of residence in a log-additive model. We also calculated odds ratios (ORs) and 95\% confidence intervals (CIs) separately for heterozygotes and rare homozygotes. The association between rs 1607237 and breast cancer risk in stage 1 of the CGEMS GWAS was evaluated as previously described (Thomas et al, 2009). Associations with breast cancer risk in the BCAC studies and the combined BCAC, MCBCS and CGEMS studies were evaluated using unconditional logistic regression adjusting for study center. A likelihood ratio test of heterogeneity by age groups was not significant $(P=0.10)$, and further adjustment for age did not change the results. Analyses of pathology-specific subsets of cases were conducted using polytomous regression with controls as the reference outcome, adjusting for study site. 
Table 2 Associations between rs 607237 and breast cancer in MCBCS, CGEMS and BCAC

2-d.f. model

\begin{tabular}{|c|c|c|c|c|c|c|}
\hline & \multirow[b]{3}{*}{ Cases } & \multirow[b]{3}{*}{ Controls } & \multirow{2}{*}{\multicolumn{2}{|c|}{ Log-additive model }} & \multirow{3}{*}{$\begin{array}{l}\text { Heterozygous } \\
\text { OR }(95 \% \mathrm{Cl})\end{array}$} & \multirow{3}{*}{$\begin{array}{l}\text { Homozygous } \\
\text { OR }(95 \% \mathrm{Cl})\end{array}$} \\
\hline & & & & & & \\
\hline & & & OR $(95 \% \mathrm{Cl})$ & $P$-value & & \\
\hline Stage 2: CGEMS & 5436 & 5280 & $0.92(0.88-0.98)$ & 0.0050 & $1.00(0.92-1.09)$ & $0.82(0.73-0.92)$ \\
\hline Stage 3: BCAC & 28766 & 28319 & $0.98(0.96-1.01)$ & 0.139 & $0.96(0.93-1.00)$ & $0.97(0.92-1.02)$ \\
\hline Combined analysis & 35991 & 35153 & $0.97(0.95-0.99)$ & 0.0046 & $0.97(0.93-1.00)$ & $0.94(0.90-0.98)$ \\
\hline Invasive & 33660 & 34988 & $0.97(0.95-0.99)$ & 0.012 & $0.97(0.94-1.00)$ & $0.95(0.90-0.99)$ \\
\hline
\end{tabular}

Abbreviations: BCAC = Breast Cancer Association Consortium; CGEMS = Cancer Genetic Markers of Susceptibility; $C l=$ confidence interval; DCIS = ductal carcinoma in situ; MCBCS = Mayo Clinic breast cancer case-control study; OR=odds ratio.

\section{RESULTS}

Of four PIK3CA haplotype-tagging SNPs, rs1607237 was significantly associated with risk of breast cancer in MCBCS $(\mathrm{OR}=0.85$, 95\% CI $0.73-0.98, P=0.023$; Table 2, Supplementary Figure 1). Next we evaluated associations between rs1607237 and breast cancer risk in 1145 cases and 1142 controls genotyped in stage 1 of the CGEMS breast cancer GWAS (Thomas et al, 2009). Rs1607237 was significantly associated with breast cancer risk (heterozygous $\mathrm{OR}=1.12$, homozygous $\mathrm{OR}=0.79$, score $P=0.017$ ). To provide a more stable estimate of risk in this population, 8429 additional CGEMS subjects were genotyped for rs1607237. In all 5436 cases and 5280 controls from stage 1 and 2 of CGEMS, rs1607237 was strongly associated with a decrease in breast cancer risk $(\mathrm{OR}=0.92,95 \%$ CI $0.88-0.98, P=0.0050$; Table 2$)$.

This finding provided the rationale for further evaluation of this SNP in 23 BCAC studies involving women of European ancestry (28766 cases, 28319 controls), and two BCAC studies of Asian women (2183 cases, 1469 controls; Table 1). Rs1607237 was not significantly associated with breast cancer risk in the 23 BCAC studies of women of European ancestry $(\mathrm{OR}=0.98,95 \%$ CI $0.96-$ $1.01, P=0.139)$ or in the two Asian BCAC studies (OR $=1.05,95 \%$ CI $0.94-1.16, P=0.39$; Table 2). However, when combining all genotype data from the three stages of this study (MCBCS, CGEMS and BCAC; Supplementary Table 3), rs1607237 was significantly associated with risk of breast cancer (OR $=0.97,95 \%$ CI $0.95-0.99$, $P=9.5 \times 10^{-3}$ ). Similarly, a significant association was observed when considering only women of European ancestry in the combined analysis $\left(\mathrm{OR}=0.97,95 \%\right.$ CI $0.95-0.99, P=4.6 \times 10^{-3}$; (Table 2). There was no evidence of heterogeneity by study site among the 25 Caucasian studies $(P=0.14$; Supplementary Figure 2).

To further understand the association with breast cancer, we restricted the analysis to women with invasive breast cancer. Rs 1607237 was associated with a reduced risk of invasive breast cancer $(\mathrm{OR}=0.97,95 \%$ CI $0.95-0.99, P=0.012$; Table 2), whereas no association with risk of ductal carcinoma in situ was observed $(\mathrm{OR}=0.93,95 \% \mathrm{CI} 0.85-1.02, P=0.12)$. In addition, we explored differences in PIK3CA SNP associations in the combined data set by tumour subtype (Supplementary Table 4). The rs1607237 variant was not associated with any subtypes defined by ER, PR or HER2 status, although it is important to note the reduction in sample size when restricting to these tumour subtypes.

\section{DISCUSSION}

Here we report an association between inherited variation in the oncogene PIK3CA and risk of breast cancer in a large, three-stage analysis utilising nearly 75000 subjects from 27 case-control study studies. We show that rs1607237 is significantly associated with a small decrease in breast cancer risk $(\mathrm{OR}=0.97,95 \% \mathrm{CI}$
0.95-0.99, $P=9.5 \times 10^{-3}$ ) in all studies combined and when considering only women of European ancestry in the combined studies $\left(\mathrm{OR}=0.97,95 \%\right.$ CI $\left.0.95-0.99, P=4.6 \times 10^{-3}\right)$. However, the association did not achieve significance in the large third stage involving only BCAC studies. Although the first two stages of our analysis suggest an association between PIK3CA and breast cancer risk, our inability to confirm this finding in the BCAC studies suggests that the result should be interpreted with caution.

We further explored the linkage disequilibrium patterns in the PIK3CA coding and promoter regions to better understand the relationship between rs1607237 and other variation in this region. Rs1607237 was not in strong linkage disequilibrium with two nonsynonymous polymorphic variants in the coding region of PIK3CA, rs1051399 $\left(r^{2}=0.0060\right)$ and $r 33729680\left(r^{2}=0.034\right)$, which had been genotyped in HapMap samples of European ancestry. However, an additional 18 non-synonymous variants were either not polymorphic or had not been genotyped in the HapMap samples, making inference about the relationship between rs1607237 and all variants of unknown significance in the PIK3CA coding region difficult. In addition, two PIK3CA promoter SNPs were in low LD with rs1607237 (rs9831234, $r^{2}=0.16$; rs2865084, $r^{2}=0.038$ ). However, it remains possible that PIK3CA promoter SNPs that were not captured in this study are related to breast cancer risk.

It is also important to note that the effect estimate for rs 1607237 in the BCAC replication studies and in the overall BCAC, MCBCS and CGEMS studies is quite small $(\mathrm{OR}=0.97)$. This limits our statistical power to detect significant associations in these studies despite the large sample size, particularly in analyses utilising pathology information that is available for only a subset of subjects. Similarly, we had limited power to detect associations in the original MCBCS study with the three non-significant PIK3CA SNPs. Thus, it remains possible that evaluation of these variants in the larger BCAC cohort might detect associations with risk. While the effect of rs1607237 on risk is small, the association between inherited variation in this important oncogene and breast cancer risk does provide valuable biological insight into the development of this disease. Validation of rs1607237 in GWAS studies from other large collaborative groups and additional studies by BCAC with detailed pathology information are necessary to confirm this association. Functional evaluation of this variant is needed to fully understand the relationship between inherited PIK3CA variation and breast cancer risk.

\section{ACKNOWLEDGEMENTS}

The ABCS study was funded by the Dutch Cancer Society (grants NKI 2001-2423; NKI 2007-3839), the Dutch National Genomics 
Initiative; ABCS acknowledges all patients, and Sten Cornelissen, Richard van Hien, Flora van Leeuwen, Vincent Smit and other contributors to the 'BOSOM' study (ABCS). The ABCFS, NC-BCFR and OFBCR works were supported by the United States National Cancer Institute, National Institutes of Health (NIH) under RFACA-06-503 and through cooperative agreements with members of the Breast Cancer Family Registry (BCFR) and Principal Investigators, including Cancer Care Ontario (U01 CA69467), Northern California Cancer Center (U01 CA69417), University of Melbourne (U01 CA69638). Samples from the NC-BCFR were processed and distributed by the Coriell Institute for Medical Research. The content of this manuscript does not necessarily reflect the views or policies of the National Cancer Institute or any of the collaborating centers in the BCFR, nor does mention of trade names, commercial products or organisations imply endorsement by the US Government or the BCFR. The ABCFS was also supported by the National Health and Medical Research Council of Australia, the New South Wales Cancer Council, the Victorian Health Promotion Foundation (Australia) and the Victorian Breast Cancer Research Consortium. JLH is a National Health and Medical Research Council (NHMRC) Australia Fellow and a Victorian Breast Cancer Research Consortium Group Leader. MCS is a NHMRC Senior Research Fellow and a Victorian Breast Cancer Research Consortium Group Leader. BBCC is funded in part by the ELAN fund of the University Hospital Erlangen. The BBCS is funded by Cancer Research UK and Breakthrough Breast Cancer and acknowledges NHS funding to the NIHR Biomedical Research Centre, and the National Cancer Research Network (NCRN). ES is supported by NIHR Comprehensive Biomedical Research Centre, Guy's \& St Thomas' NHS Foundation Trust in partnership with King's College London, United Kingdom. IT is supported by the Oxford Biomedical Research Centre. The CNIO-BCS was supported by the Genome Spain Foundation, the Red Temática de Investigación Cooperativa en Cáncer and grants from the Asociación Española Contra el Cáncer and the Fondo de Investigación Sanitario (PI081583 and PI081120). We thank Charo Alonso, Tais Moreno, Guillermo Pita, Primitiva Menéndez and Pilar Zamora for their contribution to this work. The GC-HBOC was collected within a project funded by the Deutsche Krebshilfe (Grant number: 107054). It was supported by the Dietmar-Hopp Foundation, the Helmholtz society and the German Cancer Research Center (DKFZ). We thank Sandrine Tchatchou for genotyping. The HABCS was supported by an intramural grant from Hannover Medical School. The HMBCS was supported by short-term fellowships from the German Academic Exchange Program (to NB), and the Friends of Hannover Medical School (to NB). KARBAC acknowledges The Swedish Cancer Society and The Stockholm Cancer Society. KBCP is supported by grants from the Finnish Cancer Society; the Academy of Finland (grant number 127220); the special Government Funding (EVO) of Kuopio University Hospital (grant number 5654113 and 5501) and by the strategic funding of the University of Eastern Finland. We thank Mrs Helena Kemiläinen, Mrs Aija Parkkinen and Mrs Eija Myöhänen for their skillful technical assistance. We thank Heather Thorne, Eveline Niedermayr, all the kConFab research nurses and staff, the heads and staff of the Family Cancer Clinics, and the Clinical Follow Up Study (funded 2001-2009 by NHMRC and currently by the National Breast Cancer Foundation and Cancer Australia \#628333) for their contributions to this resource, and the many families who contribute to kConFab. kConFab is supported by grants from the National Breast Cancer Foundation, the National Health and Medical Research Council (NHMRC) and by the Queensland Cancer Fund, the Cancer Councils of New South Wales, Victoria, Tasmania and South Australia, and the Cancer Foundation of Western Australia Financial support for the AOCS was provided by the United States Army Medical Research and Materiel Command (DAMD17-01-1-0729); the Cancer Council of Tasmania and Cancer Foundation of Western Australia; and the NHMRC [199600]. GC-T is supported by the NHMRC. PP is supported by funds from Italian citizens who allocated the $5 \times 1000$ share of their tax payment to the Fondazione IRCCS Istituto Nazionale Tumori, according to Italian laws (INTInstitutional strategic projects ' $5 \times 1000$ '). MBCSG thanks Paolo Radice, Bernard Peissel, Daniela Zaffaroni and Marco A Pierotti of the Fondazione IRCCS Istituto Nazionale Tumori and Monica Barile of the Istituto Europeo di Oncologia, Milano, Italy. MCBCS was supported by NIH grant CA122340 and US Recovery act award CA122340Z. Many people have contributed to the MCCS, including the original investigators and the diligent teams who recruited the participants and who continue working on follow-up. Finally, we express our gratitude to the many thousands of Melbourne residents who continue to participate in the study. Cohort recruitment was funded by VicHealth and Cancer Council Victoria. The MCCS was further supported by Australian NHMRC grants 209057, 251553 and 504711 and by infrastructure provided by Cancer Council Victoria. OBCS was supported by research grants from the Finnish Cancer Foundation, the Sigrid Juselius Foundation, the Academy of Finland, the University of Oulu and the Oulu University Hospital. SBCS was funded by the Breast Cancer Campaign and Yorkshire Cancer Research. The authors acknowledge Helen Cramp, Sue Higham, Dan Connley, Saba Balasubramanian. The UCIBCS is supported by the National Institutes of Health, National Cancer Institute grants CA-58860, CA-92044 and the Lon V Smith Foundation grant LVS-39420.

\section{Conflict of interest}

The authors declare no conflict of interest.

Supplementary Information accompanies the paper on British Journal of Cancer website (http://www.nature.com/bjc)

\section{REFERENCES}

Aleskandarany MA, Rakha EA, Ahmed MA, Powe DG, Paish EC, Macmillan RD, Ellis IO, Green AR (2010) PIK3CA expression in invasive breast cancer: a biomarker of poor prognosis. Breast Cancer Res Treat 122(1): $45-53$

Bachman KE, Argani P, Samuels Y, Silliman N, Ptak J, Szabo S, Konishi H, Karakas B, Blair BG, Lin C, Peters BA, Velculescu VE, Park BH (2004) The PIK3CA gene is mutated with high frequency in human breast cancers. Cancer Biol Ther 3(8): 772-775

Campbell IG, Russell SE, Choong DY, Montgomery KG, Ciavarella ML, Hooi CS, Cristiano BE, Pearson RB, Phillips WA (2004) Mutation of the PIK3CA gene in ovarian and breast cancer. Cancer Res 64(21): 7678-7681

Cantley LC (2002) The phosphoinositide 3-kinase pathway. Science 296(5573): $1655-1657$
Fresno Vara JA, Casado E, de Castro J, Cejas P, Belda-Iniesta C, Gonzalez-Baron M (2004) PI3K/Akt signalling pathway and cancer. Cancer Treat Rev 30(2): 193-204

Fruman DA, Meyers RE, Cantley LC (1998) Phosphoinositide kinases. Annu Rev Biochem 67: $481-507$

Garcia-Closas M, Hall P, Nevanlinna H, Pooley K, Morrison J, Richesson DA, Bojesen SE, Nordestgaard BG, Axelsson CK, Arias JI, Milne RL, Ribas G, Gonzalez-Neira A, Benitez J, Zamora P, Brauch H, Justenhoven C, Hamann U, Ko YD, Bruening T, Haas S, Dork T, Schurmann P, Hillemanns P, Bogdanova N, Bremer M, Karstens JH, Fagerholm R, Aaltonen K, Aittomaki K, von Smitten K, Blomqvist C, Mannermaa A, Uusitupa M, Eskelinen M, Tengstrom M, Kosma VM, Kataja V, Chenevix-Trench G, Spurdle AB, Beesley J, Chen X, Devilee P, van 
Asperen CJ, Jacobi CE, Tollenaar RA, Huijts PE, Klijn JG, Chang-Claude J, Kropp S, Slanger T, Flesch-Janys D, Mutschelknauss E, Salazar R, WangGohrke S, Couch F, Goode EL, Olson JE, Vachon C, Fredericksen ZS, Giles GG, Baglietto L, Severi G, Hopper JL, English DR, Southey MC, Haiman CA, Henderson BE, Kolonel LN, Le Marchand L, Stram DO, Hunter DJ, Hankinson SE, Cox DG, Tamimi R, Kraft P, Sherman ME, Chanock SJ, Lissowska J, Brinton LA, Peplonska B, Hooning MJ, MeijersHeijboer H, Collee JM, van den Ouweland A, Uitterlinden AG, Liu J, Lin LY, Yuqing L, Humphreys K, Czene K, Cox A, Balasubramanian SP, Cross SS, Reed MW, Blows F, Driver K, Dunning A, Tyrer J, Ponder BA, Sangrajrang S, Brennan P, McKay J, Odefrey F, Gabrieau V, Sigurdson A, Doody M, Struewing JP, Alexander B, Easton DF, Pharoah PD (2008) Heterogeneity of breast cancer associations with five susceptibility loci by clinical and pathological characteristics. PLoS Genet 4(4): e1000054

Kalinsky K, Jacks LM, Heguy A, Patil S, Drobnjak M, Bhanot UK, Hedvat CV, Traina TA, Solit D, Gerald W, Moynahan ME (2009) PIK3CA mutation associates with improved outcome in breast cancer. Clin Cancer Res 15(16): 5049-5059

Karakas B, Bachman KE, Park BH (2006) Mutation of the PIK3CA oncogene in human cancers. Br J Cancer 94(4): 455-459

Lai YL, Mau BL, Cheng WH, Chen HM, Chiu HH, Tzen CY (2008) PIK3CA exon 20 mutation is independently associated with a poor prognosis in breast cancer patients. Ann Surg Oncol 15(4): 1064-1069

Li SY, Rong M, Grieu F, Iacopetta B (2006) PIK3CA mutations in breast cancer are associated with poor outcome. Breast Cancer Res Treat 96(1): 91 -95

Ligresti G, Militello L, Steelman LS, Cavallaro A, Basile F, Nicoletti F, Stivala F, McCubrey JA, Libra M (2009) PIK3CA mutations in human solid tumors: role in sensitivity to various therapeutic approaches. Cell Cycle 8(9): $1352-1358$

Loi S, Haibe-Kains B, Majjaj S, Lallemand F, Durbecq V, Larsimont D, Gonzalez-Angulo AM, Pusztai L, Symmans WF, Bardelli A, Ellis P, Tutt AN, Gillett CE, Hennessy BT, Mills GB, Phillips WA, Piccart MJ, Speed TP, McArthur GA, Sotiriou C (2010) PIK3CA mutations associated with gene signature of low $\mathrm{mTORC1}$ signaling and better outcomes in estrogen receptorpositive breast cancer. Proc Natl Acad Sci USA 107(22): 10208-10213

Ma YY, Wei SJ, Lin YC, Lung JC, Chang TC, Whang-Peng J, Liu JM, Yang DM, Yang WK, Shen CY (2000) PIK3CA as an oncogene in cervical cancer. Oncogene 19(23): 2739-2744

Perez-Tenorio G, Alkhori L, Olsson B, Waltersson MA, Nordenskjold B, Rutqvist LE, Skoog L, Stal O (2007) PIK3CA mutations and PTEN loss correlate with similar prognostic factors and are not mutually exclusive in breast cancer. Clin Cancer Res 13(12): 3577-3584

Saal LH, Holm K, Maurer M, Memeo L, Su T, Wang X, Yu JS, Malmstrom PO, Mansukhani M, Enoksson J, Hibshoosh H, Borg A, Parsons R (2005) PIK3CA mutations correlate with hormone receptors, node metastasis, and ERBB2, and are mutually exclusive with PTEN loss in human breast carcinoma. Cancer Res 65(7): 2554-2559

Samuels Y, Ericson K (2006) Oncogenic PI3K and its role in cancer. Curr Opin Oncol 18(1): $77-82$

Samuels Y, Wang Z, Bardelli A, Silliman N, Ptak J, Szabo S, Yan H, Gazdar A, Powell SM, Riggins GJ, Willson JK, Markowitz S, Kinzler KW, Vogelstein B, Velculescu VE (2004) High frequency of mutations of the PIK3CA gene in human cancers. Science 304(5670): 554

Shayesteh L, Lu Y, Kuo WL, Baldocchi R, Godfrey T, Collins C, Pinkel D, Powell B, Mills GB, Gray JW (1999) PIK3CA is implicated as an oncogene in ovarian cancer. Nat Genet 21(1): 99-102

\section{APPENDIX}

\section{The GENICA Network}

Gene Environment Interaction and Breast Cancer in Germany (GENICA): Dr. Margarete Fischer-Bosch-Institute of Clinical Pharmacology, Stuttgart, and University Tübingen, Germany (Hiltrud Brauch, Christina Justenhoven); Molecular Genetics of Breast Cancer, Deutsches Krebsforschungszentrum (DKFZ), Heidelberg, Germany (UH); Department of Internal Medicine, Evangelische Kliniken Bonn gGmbH, Johanniter Krankenhaus, Bonn, Germany (YDK, Christian Baisch); Institute of Pathology, Medical Faculty of the University of Bonn, Germany (Hans-Peter Fischer); Institute for Prevention and Occupational Medicine of
Stemke-Hale K, Gonzalez-Angulo AM, Lluch A, Neve RM, Kuo WL, Davies M, Carey M, Hu Z, Guan Y, Sahin A, Symmans WF, Pusztai L, Nolden LK, Horlings H, Berns K, Hung MC, van de Vijver MJ, Valero V, Gray JW, Bernards R, Mills GB, Hennessy BT (2008) An integrative genomic and proteomic analysis of PIK3CA, PTEN, and AKT mutations in breast cancer. Cancer Res 68(15): 6084-6091

Thomas G, Jacobs KB, Kraft P, Yeager M, Wacholder S, Cox DG, Hankinson SE, Hutchinson A, Wang Z, Yu K, Chatterjee N, Garcia-Closas M, Gonzalez-Bosquet J, Prokunina-Olsson L, Orr N, Willett WC, Colditz GA, Ziegler RG, Berg CD, Buys SS, McCarty CA, Feigelson HS, Calle EE, Thun MJ, Diver R, Prentice R, Jackson R, Kooperberg C, Chlebowski R, Lissowska J, Peplonska B, Brinton LA, Sigurdson A, Doody M, Bhatti P, Alexander BH, Buring J, Lee IM, Vatten LJ, Hveem K, Kumle M, Hayes RB, Tucker M, Gerhard DS, Fraumeni Jr JF, Hoover RN, Chanock SJ, Hunter DJ (2009) A multistage genome-wide association study in breast cancer identifies two new risk alleles at 1p11.2 and 14q24.1 (RAD51L1). Nat Genet 41(5): $579-584$

Wang X, Goode EL, Fredericksen ZS, Vierkant RA, Pankratz VS, Liu-Mares W, Rider DN, Vachon CM, Cerhan JR, Olson JE, Couch FJ (2008) Association of genetic variation in genes implicated in the beta-catenin destruction complex with risk of breast cancer. Cancer Epidemiol Biomarkers Prev 17(8): 2101-2108

Yang XR, Chang-Claude J, Goode EL, Couch FJ, Nevanlinna H, Milne RL, Gaudet M, Schmidt MK, Broeks A, Cox A, Fasching PA, Hein R, Spurdle AB, Blows F, Driver K, Flesch-Janys D, Heinz J, Sinn P, Vrieling A, Heikkinen T, Aittomaki K, Heikkila P, Blomqvist C, Lissowska J, Peplonska B, Chanock S, Figueroa J, Brinton L, Hall P, Czene K, Humphreys K, Darabi H, Liu J, Van 't Veer LJ, van Leeuwen FE, Andrulis IL, Glendon G, Knight JA, Mulligan AM, O’Malley FP, Weerasooriya N, John EM, Beckmann MW, Hartmann A, Weihbrecht SB, Wachter DL, Jud SM, Loehberg CR, Baglietto L, English DR, Giles GG, McLean CA, Severi G, Lambrechts D, Vandorpe T, Weltens C, Paridaens R, Smeets A, Neven P, Wildiers H, Wang X, Olson JE, Cafourek V, Fredericksen Z, Kosel M, Vachon C, Cramp HE, Connley D, Cross SS, Balasubramanian SP, Reed MW, Dork T, Bremer M, Meyer A, Karstens JH, Ay A, ParkSimon TW, Hillemanns P, Arias Perez JI, Menendez Rodriguez P, Zamora P, Benitez J, Ko YD, Fischer HP, Hamann U, Pesch B, Bruning T, Justenhoven C, Brauch H, Eccles DM, Tapper WJ, Gerty SM, Sawyer EJ, Tomlinson IP, Jones A, Kerin M, Miller N, McInerney N, Anton-Culver H, Ziogas A, Shen CY, Hsiung CN, Wu PE, Yang SL, Yu JC, Chen ST, Hsu GC, Haiman CA, Henderson BE, Le Marchand L, Kolonel LN, Lindblom A, Margolin S, Jakubowska A, Lubinski J, Huzarski T, Byrski T, Gorski B, Gronwald J, Hooning MJ, Hollestelle A, van den Ouweland AM, Jager A, Kriege M, Tilanus-Linthorst MM, Collee M, Wang-Gohrke S, Pylkas K, Jukkola-Vuorinen A, Mononen K, Grip M, Hirvikoski P, Winqvist R, Mannermaa A, Kosma VM, Kauppinen J, Kataja V, Auvinen P, Soini Y, Sironen R, Bojesen SE, Orsted DD, Kaur-Knudsen D, Flyger H, Nordestgaard BG, Holland H, Chenevix-Trench G, Manoukian S, Barile M, Radice P, Hankinson SE, Hunter DJ, Tamimi R, Sangrajrang S, Brennan P, McKay J, Odefrey F, Gaborieau V, Devilee P, Huijts PE, Tollenaar RA, Seynaeve C, Dite GS, Apicella C, Hopper JL, Hammet F, Tsimiklis H, Smith LD, Southey MC, Humphreys MK, Easton D, Pharoah P, Sherman ME, Garcia-Closas M (2011) Associations of breast cancer risk factors with tumor subtypes: a pooled analysis from the Breast Cancer Association Consortium studies. J Natl Cancer Inst 103(3): $250-263$

the German Social Accident Insurance (IPA), Bochum, Germany (TB, Beate Pesch, Volker Harth, Sylvia Rabstein). The GENICA was funded by the Federal Ministry of Education and Research (BMBF) Germany grants 01KW9975/5, 01KW9976/8, 01KW9977/0 and 01KW0114, the Robert Bosch Foundation, Stuttgart, Deutsches Krebsforschungszentrum (DKFZ), Heidelberg, Institute for Prevention and Occupational Medicine of the German Social Accident Insurance (IPA), Bochum, as well as the Department of Internal Medicine, Evangelische Kliniken Bonn gGmbH, Johanniter Krankenhaus, Bonn, Germany.

kConFab Investigators, Australian Ovarian Cancer Study Group Peter MacCallum Cancer Centre, St Andrews Place, East Melbourne VIC 3002, Australia.

This work is published under the standard license to publish agreement. After 12 months the work will become freely available and the license terms will switch to a Creative Commons Attribution-NonCommercial-Share Alike 3.0 Unported License. 